\title{
COPRIME ARRAYS AND SAMPLERS FOR SPACE-TIME ADAPTIVE PROCESSING
}

\author{
Chun-Lin Liu $^{1}$ and P. P. Vaidyanathan ${ }^{2}$ \\ Dept. of Electrical Engineering, 136-93 \\ California Institute of Technology, Pasadena, CA 91125, USA \\ E-mail: cl.liu@ caltech.edu ${ }^{1}$ and ppvnath@systems.caltech.edu ${ }^{2}$
}

\begin{abstract}
This paper extends the use of coprime arrays and samplers for the case of moving sources. Space-time adaptive processing (STAP) plays an important role in estimating direction-of-arrivals (DOAs) and radial velocities of emitting sources. However, the detection performance is fundamentally limited by the array geometry and the temporal samplers at each sensor. Coprime arrays and coprime samplers offer an enhanced degree of freedom of $O(M N)$ using only $O(M+N)$ physical sensors or samples. In this paper, we propose coprime joint angle-Doppler estimation (coprime JADE), which incorporates both coprime arrays and coprime samplers with the STAP framework. Nonuniform time samples at different sensors can be used to generate a sampled autocorrelation matrix, from which we compute a spatial smoothed matrix. It will be proved that spatial smoothed matrices can be used in the MUSIC algorithm for parameter estimation. With sufficient snapshots, coprime JADE distinguishes $O\left(M_{1} N_{1} M_{2} N_{2}\right)$ independent sources if it corresponds to coprime arrays and coprime samplers with coprime integers $\left(M_{1}, N_{1}\right)$ and $\left(M_{2}, N_{2}\right)$, respectively. It is verified through simulations that coprime JADE resolves the angle-Doppler information better compared to other conventional algorithms.
\end{abstract}

Index Terms - Coprime arrays, coprime samplers, joint angleDoppler estimation, the MUSIC algorithm.

\section{INTRODUCTION}

Space-time adaptive processing (STAP) is a standardized technique in airbone MTI radar signal processing [1,2]. From time samples at different sensors, adaptive weightings are applied to these data to derive a $2 \mathrm{D}$ beamformer for direction-of-arrivals (DOAs) and Doppler frequencies. Estimators based on power spectrum density (PSD) [1], minimum variance distortionless response (MVDR) [3], MUSIC algorithms [4], ESPRIT algorithms [5], time-space-time MUSIC [6], and compressive joint angular-frequency power spectrum estimation [7] are also popular in extracting the angle-Doppler information.

Coprime arrays have received attention in DOA estimation since they can resolve more sources than the number of sensors [8]. Consisting of two uniform linear arrays (ULAs) whose interelement spacings are $M d$ and $N d$, coprime arrays provide an enhanced degree of freedom of $O(M N)$ using $O(M+N)$ sensors, assuming $M$ and $N$ are coprime integers. This property facilitates superresolution applications in DOA estimation $[9,10]$, spectrum sensing [8], and other applications.

In this paper, we propose a novel joint angle-Doppler estimation framework using coprime arrays for sensors and coprime samplers at

This work was supported in parts by the ONR grant N00014-11-1-0676, and the California Institute of Technology. the output of each sensor. This will be called coprime JADE in this paper. Designing coprime arrays with coprime integers $M_{1}$ and $N_{1}$ in the spatial domain and coprime samplers with coprime integers $M_{2}$ and $N_{2}$ in the time domain enables superresolution on the angleDoppler plane. By using coprime arrays and samplers, it will be proved that coprime JADE can identify $O\left(M_{1} N_{1} M_{2} N_{2}\right)$ distinct sources.

This paper is organized as follows. In Section 2, the data model is briefly reviewed. In Section 3, coprime JADE is introduced in detail. In Section 4, we propose identifiability guarantees for coprime JADE, which are verified by simulations in Section 5 before Section 6 concludes this paper.

\section{THE DATA MODEL}

Consider $D$ sources emitting electromagnetic wave in the space. Our array receives the emitted signals at sensor locations $n d$ in space, and then takes samples at time instant $m T$ in time, where $d=\lambda / 2$ denotes the minimum spacing between sensors and $T$ is the minimum sampling interval. Each source is characterized by its DOA $\theta_{i}$ and its radial velocity $v_{i}$. The received signal $X(n, m)$ can be modelled as a random variable defined by

$$
X(n, m)=\sum_{i=1}^{D} A_{i} e^{j 2 \pi \bar{\theta}_{i} n} e^{j 2 \pi \bar{f}_{i} m}+N(n, m),
$$

where $\left\{A_{i}\right\}_{i=1}^{D}$ are zero-mean random variables with the uncorrelated property $\mathbb{E}\left[A_{i}^{*} A_{j}\right]=\sigma_{i}^{2} \delta_{i, j}$. Here $\bar{\theta}_{i}$ and $\bar{f}_{i}$ are normalized DOAs and normalized Doppler frequencies defined by

$$
\bar{\theta}_{i}=\frac{d}{\lambda} \sin \theta_{i}, \quad \bar{f}_{i}=\frac{T}{\lambda} v_{i},
$$

where $\bar{\theta}_{i}, \bar{f}_{i} \in[-1 / 2,1 / 2) . N(n, m)$ stand for additive white noise with $\mathbb{E}\left[N^{*}(n, m) N\left(n^{\prime}, m^{\prime}\right)\right]=\sigma^{2} \delta_{n, n^{\prime}} \delta_{m, m^{\prime}}$. Noise is assumed uncorrelated with the sources $\left\{A_{i}\right\}_{i=1}^{D}$.

Eq. (1) can be reformulated using matrices. Defining a random matrix $\mathbf{X}=[X(n, m)]$, Eq. (1) becomes

$$
\mathbf{X}=\sum_{i=1}^{D} A_{i} \mathbf{v}_{s}\left(\bar{\theta}_{i}\right) \mathbf{v}_{t}^{T}\left(\bar{f}_{i}\right)+\mathbf{N},
$$

where $\mathbf{v}_{s}\left(\bar{\theta}_{i}\right)$ denote spatial steering vectors corresponding to $\bar{\theta}_{i}$, and $\mathbf{v}_{t}\left(\bar{f}_{i}\right)$ are temporal steering vectors with respect to normalized Doppler frequencies $\bar{f}_{i}$. The superscript $T$ stands for matrix transpose operation and $\mathbf{N}=[N(n, m)]$. Vectorizing (2) leads to the following expression

$$
\mathbf{x}=\sum_{i=1}^{D} A_{i} \mathbf{v}_{s, t}\left(\bar{\theta}_{i}, \bar{f}_{i}\right)+\mathbf{n}
$$




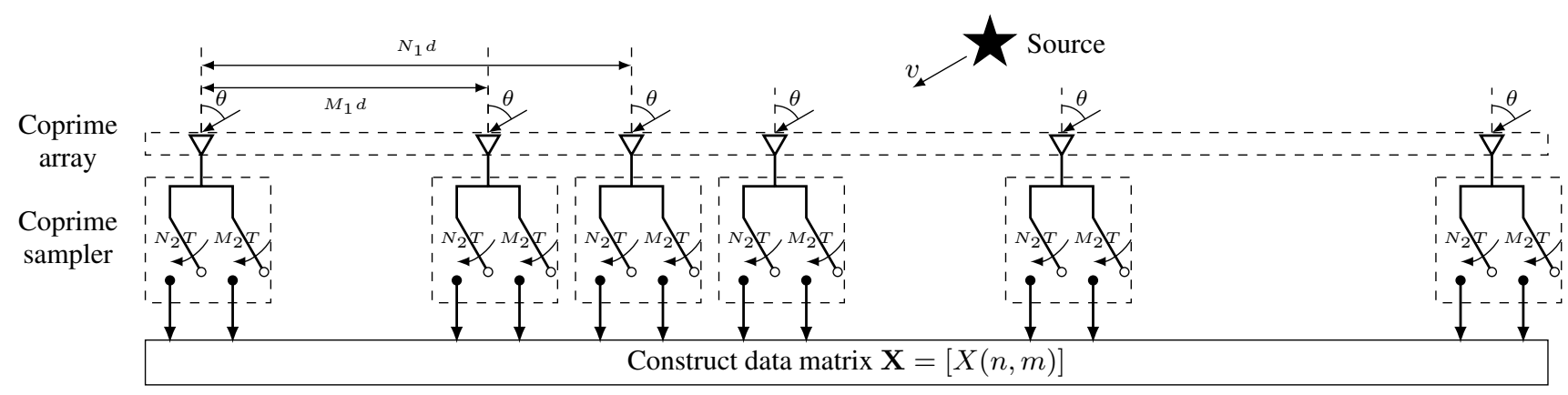

Fig. 1. A system diagram of coprime JADE.

where $\mathbf{x}=\operatorname{vec}(\mathbf{X}), \mathbf{n}=\operatorname{vec}(\mathbf{N})$, and

$$
\mathbf{v}_{s, t}\left(\bar{\theta}_{i}, \bar{f}_{i}\right)=\mathbf{v}_{t}\left(\bar{f}_{i}\right) \otimes \mathbf{v}_{s}\left(\bar{\theta}_{i}\right)
$$

represents space-time steering vector related to the angle-Doppler frequency pair $\left(\bar{\theta}_{i}, \bar{f}_{i}\right)$. Here $\otimes$ is the Kronecker product between matrices.

In practice, multiple snapshots are taken to estimate second order statistics if sources are stationary within these snapshots. Assume these multiple measurements are written as $\mathbf{x}(k)$, where $k=$ $1,2, \ldots, K$. The autocorrelation matrix of $\mathbf{x}$ can be estimated as

$$
\hat{\mathbf{R}}_{\mathbf{x}}=\frac{1}{K} \sum_{k=1}^{K} \mathbf{x}(k) \mathbf{x}^{H}(k),
$$

where the superscript $H$ stands for transpose-conjugate of matrices. The estimated autocorrelation matrix can then be applied to beamformers, MUSIC algorithms, or coprime arrays/samplers.

\section{JOINT ANGLE-DOPPLER ESTIMATION}

In this section, we present a novel JADE setting using coprime arrays and coprime samplers to achieve much higher degree of freedom. From the coprime samples, the autocorrelation matrix is first estimated. Based on the difference set pertaining to coprime arrays and coprime samplers, the spatial smoothing method is applied to the sampled autocorrelation matrix, yielding a larger-size spatial smoothed matrix. Then the MUSIC algorithm is applied to estimate the angle-Doppler information.

Fig. 1 depicts the system diagram of coprime JADE. The coprime array is composed of two uniform linear arrays with interelement spacing $M_{1} d$ and $N_{1} d$, where $M_{1}$ and $N_{1}$ are coprime integers. The sensor locations are explicitly defined over the grid $n d$, where

$$
n \in\left\{0, M_{1}, \ldots,\left(N_{1}-1\right) M_{1}, N_{1}, 2 N_{1}, \ldots,\left(2 M_{1}-1\right) N_{1}\right\} .
$$

There are $N_{1}+2 M_{1}-1$ sensors in total. At each sensor, two samplers operate at sampling rates $M_{2} T$ and $N_{2} T$ in parallel to obtain coprime samples, where $M_{2}$ and $N_{2}$ are also coprime integers. These time-domain samples are defined at time instant $m T$, where

$$
m \in\left\{0, M_{2}, \ldots,\left(N_{2}-1\right) M_{2}, N_{2}, 2 N_{2}, \ldots,\left(2 M_{2}-1\right) N_{2}\right\} .
$$

Each sampler takes $N_{2}+2 M_{2}-1$ samples per sensor in one snapshot.

Next, the autocorrelation matrix of $\mathbf{x}$ is studied. From (3), the ideal autocorrelation matrix of $\mathbf{x}$ is found to be

$$
\mathbb{E}\left[\mathbf{x x}^{H}\right]=\sum_{i=1}^{D} \sigma_{i}^{2} \mathbf{v}_{s, t}\left(\bar{\theta}_{i}, \bar{f}_{i}\right) \mathbf{v}_{s, t}^{H}\left(\bar{\theta}_{i}, \bar{f}_{i}\right)+\sigma^{2} \mathbf{I} .
$$

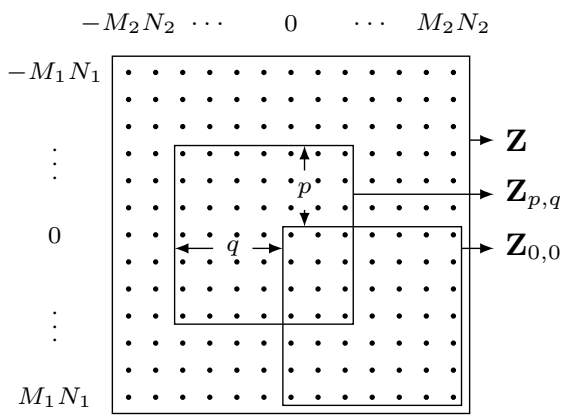

Fig. 2. Relationships between $\mathbf{Z}$ and its submatrix $\mathbf{Z}_{p, q}$.

The term $\mathbf{v}_{s, t}\left(\bar{\theta}_{i}, \bar{f}_{i}\right) \mathbf{v}_{s, t}^{H}\left(\bar{\theta}_{i}, \bar{f}_{i}\right)$ can be rewritten as follows

$$
\left(\mathbf{v}_{t}\left(\bar{f}_{i}\right) \mathbf{v}_{t}^{H}\left(\bar{f}_{i}\right)\right) \otimes\left(\mathbf{v}_{s}\left(\bar{\theta}_{i}\right) \mathbf{v}_{s}^{H}\left(\bar{\theta}_{i}\right)\right),
$$

which is because of (4) and the property $(\mathbf{A} \otimes \mathbf{B})(\mathbf{C} \otimes \mathbf{D})=$ $(\mathbf{A C}) \otimes(\mathbf{B D})$. Note that the entries in $\mathbf{v}_{s}\left(\bar{\theta}_{i}\right) \mathbf{v}_{s}^{H}\left(\bar{\theta}_{i}\right)$ are in the form of $e^{j 2 \pi \bar{\theta}_{i} \tilde{n}}$, where the range set of $\tilde{n}$ contains all integers from $-M_{1} N_{1}$ to $M_{1} N_{1}$ [9]. Hence, there exists an arrangement that converts $\mathbf{v}_{s}\left(\bar{\theta}_{i}\right) \mathbf{v}_{s}^{H}\left(\bar{\theta}_{i}\right)$ to a new steering vector $\mathbf{w}_{s}\left(\bar{\theta}_{i}\right)=\left[e^{j 2 \pi \bar{\theta}_{i} \tilde{n}}\right]$, where $\tilde{n}=-M_{1} N_{1}, \ldots, M_{1} N_{1}$. $\mathbf{w}_{s}\left(\bar{\theta}_{i}\right)$ can be also regarded as steering vectors corresponding to a $\left(2 M_{1} N_{1}+1\right)$-element ULA in coarray domain. The same argument holds true in the time domain, where a new steering vector $\mathbf{w}_{t}\left(\bar{f}_{i}\right)$ is viewed as the collection of $2 M_{2} N_{2}+1$ uniform samples. According to the equivalence of (2) and (3), a matrix $\mathbf{Z} \in \mathbb{C}^{\left(2 M_{1} N_{1}+1\right) \times\left(2 M_{2} N_{2}+1\right)}$ is constructed from $\mathbb{E}\left[\mathbf{x x}^{H}\right]$ such that

$$
\mathbf{Z}=\sum_{i=1}^{D} \sigma_{i}^{2} \mathbf{w}_{s}\left(\bar{\theta}_{i}\right) \mathbf{w}_{t}^{T}\left(\bar{f}_{i}\right)+\sigma^{2} \mathbf{e}_{1} \mathbf{e}_{2}^{T},
$$

where $\mathbf{e}_{i}=\left[\delta_{\ell, M_{i} N_{i}+1}\right]_{\ell=1}^{2 M_{i} N_{i}+1}$ for $i=1,2$. Eq. (6) can be interpreted as a sum of $D$ coherent sources in $2 D$. Then, for coherent sources, spatial smoothing [11-13] is a technique for estimating the autocorrelation matrix of $\mathbf{Z}$, from which the MUSIC algorithm [4] can resolve the DOA and the Doppler frequency information.

To apply spatial smoothing, the submatrix of $\mathbf{Z}$ is defined as $\mathbf{Z}_{p, q} \in \mathbb{C}^{\left(M_{1} N_{1}+1\right) \times\left(M_{2} N_{2}+1\right)}$, which is depicted in Fig. 2,

$$
\mathbf{Z}_{p, q}=\sum_{i=1}^{D} \sigma_{i}^{2} e^{-j 2 \pi\left(\bar{\theta}_{i} p+\bar{f}_{i} q\right)} \tilde{\mathbf{w}}_{s}\left(\bar{\theta}_{i}\right) \tilde{\mathbf{w}}_{t}^{T}\left(\bar{f}_{i}\right)+\sigma^{2} \mathbf{e}_{1, p} \mathbf{e}_{2, q}^{T},
$$


where $\tilde{\mathbf{w}}_{s}\left(\bar{\theta}_{i}\right) \in \mathbb{C}^{M_{1} N_{1}+1}$ and $\tilde{\mathbf{w}}_{t}\left(\bar{f}_{i}\right) \in \mathbb{C}^{M_{2} N_{2}+1}$ are space and time steering vectors of an ULA and uniform sampling starting with 0 , respectively. $\mathbf{e}_{1, p}$ is a subvector constructed from the $\left(M_{1} N_{1}+\right.$ $1-p)$ th entry to the $\left(2 M_{1} N_{1}+1-p\right)$ th entry of $\mathbf{e}_{1}$. Here $p \in$ $\left\{0,1, \ldots, M_{1} N_{1}\right\}, q \in\left\{0,1, \ldots, M_{2} N_{2}\right\}$.

We then define the spatial smoothed matrix $\mathbf{R}_{s s}$ as

$$
\mathbf{R}_{s s}=\frac{1}{\left(M_{1} N_{1}+1\right)\left(M_{2} N_{2}+1\right)} \sum_{p=0}^{M_{1} N_{1}} \sum_{q=0}^{M_{2} N_{2}} \mathbf{z}_{p, q} \mathbf{z}_{p, q}^{H},
$$

where $\mathbf{z}_{p, q}=\operatorname{vec}\left(\mathbf{Z}_{p, q}\right)$. It follows from (7) that $\mathbf{R}_{s s}$ is a Hermitian, positive semidefinite matrix of size $\left(M_{1} N_{1}+1\right)\left(M_{2} N_{2}+1\right)$ by- $\left(M_{1} N_{1}+1\right)\left(M_{2} N_{2}+1\right)$.

Theorem 1. The spatial smoothed matrix $\mathbf{R}_{s s}$ can be expressed as

$$
\mathbf{R}_{s s}=C^{2}\left(\sum_{i=1}^{D} \sigma_{i}^{2} \tilde{\mathbf{w}}_{s, t}\left(\bar{\theta}_{i}, \bar{f}_{i}\right) \tilde{\mathbf{w}}_{s, t}^{H}\left(\bar{\theta}_{i}, \bar{f}_{i}\right)+\sigma^{2} \mathbf{I}\right)^{2},
$$

where $\tilde{\mathbf{w}}_{s, t}\left(\bar{\theta}_{i}, \bar{f}_{i}\right)=\tilde{\mathbf{w}}_{t}\left(\bar{f}_{i}\right) \otimes \tilde{\mathbf{w}}_{s}\left(\bar{\theta}_{i}\right)$ are space-time steering vectors, and $C=\left(\left(M_{1} N_{1}+1\right)\left(M_{2} N_{2}+1\right)\right)^{-1 / 2}$.

Proof. The proof is sketched as follows. Analogous to (2) and (3), $\mathbf{z}_{p, q}=\operatorname{vec}\left(\mathbf{Z}_{p, q}\right)$ can be written in terms of $\tilde{\mathbf{w}}_{s, t}\left(\bar{\theta}_{i}, \bar{f}_{i}\right)$. Then, putting $\mathbf{z}_{p, q}$ into (7) leads to an expression in terms of summations over $p, q, i_{1}, i_{2}$, where $i_{1}$ and $i_{2}$ are indices which run over all sources. The cross terms in $\mathbf{R}_{s s}$ are replaced with steering vectors,

$$
\begin{aligned}
\tilde{\mathbf{w}}_{s}^{H}\left(\bar{\theta}_{i_{1}}\right) \tilde{\mathbf{w}}_{s}\left(\bar{\theta}_{i_{2}}\right) & =\sum_{p=0}^{M_{1} N_{1}} e^{-j 2 \pi \bar{\theta}_{i_{1}} p} e^{j 2 \pi \bar{\theta}_{i_{2}} p}, \\
\tilde{\mathbf{w}}_{t}^{H}\left(\bar{f}_{i_{1}}\right) \tilde{\mathbf{w}}_{t}\left(\bar{f}_{i_{2}}\right) & =\sum_{q=0}^{M_{2} N_{2}} e^{-j 2 \pi \bar{f}_{i_{1}} q} e^{j 2 \pi \bar{f}_{i_{2}} q}, \\
\tilde{\mathbf{w}}_{t}\left(\bar{f}_{i_{2}}\right) \otimes \tilde{\mathbf{w}}_{s}\left(\bar{\theta}_{i_{2}}\right) & =\sum_{p=0}^{M_{1} N_{1}} \sum_{q=0}^{M_{2} N_{2}} e^{j 2 \pi\left(\bar{\theta}_{i_{2}} p+\bar{f}_{i_{2}} q\right)}\left(\mathbf{e}_{2, q} \otimes \mathbf{e}_{1, p}\right) .
\end{aligned}
$$

Then $\mathbf{R}_{s s} / C^{2}$ can be expressed as

$$
\begin{aligned}
& \sum_{i_{1}=1}^{D} \sum_{i_{2}=1}^{D}\left[\sigma_{i_{1}}^{2} \sigma_{i_{2}}^{2}\left(\tilde{\mathbf{w}}_{t}\left(\bar{f}_{i_{1}}\right) \tilde{\mathbf{w}}_{t}^{H}\left(\bar{f}_{i_{1}}\right) \tilde{\mathbf{w}}_{t}\left(\bar{f}_{i_{2}}\right) \tilde{\mathbf{w}}_{t}^{H}\left(\bar{f}_{i_{2}}\right)\right)\right. \\
& \left.\otimes\left(\tilde{\mathbf{w}}_{s}\left(\bar{\theta}_{i_{1}}\right) \tilde{\mathbf{w}}_{s}^{H}\left(\bar{\theta}_{i_{1}}\right) \tilde{\mathbf{w}}_{s}\left(\bar{\theta}_{i_{2}}\right) \tilde{\mathbf{w}}_{s}^{H}\left(\bar{\theta}_{i_{2}}\right)\right)\right] \\
& +2 \sum_{i=1}^{D} \sigma_{i}^{2} \sigma^{2} \tilde{\mathbf{w}}_{s, t}\left(\bar{\theta}_{i}, \bar{f}_{i}\right) \tilde{\mathbf{w}}_{s, t}^{H}\left(\bar{\theta}_{i}, \bar{f}_{i}\right)+\sigma^{4} \mathbf{I} .
\end{aligned}
$$

Rearranging (9) proves this theorem.

Note that the theorems similar to Theorem 1 have already been proposed in the context of 1D coprime arrays [9], 1D nested arrays [13], and 2D nested arrays [14]. It is also observed that Theorem 1 resembles Theorem 3 of [14] because coprime JADE follows the same formulation as coprime arrays in two dimensions [15] and nested arrays in two dimensions [14]. In other words, our work bridges the gap between STAP and two dimensional array processing. The former estimates the DOA and the Doppler frequency while the latter seeks the azimuth and the elevation in two dimensions.

However, coprime JADE and coprime arrays in 2D are related to different design problems. In coprime JADE, sensor locations are fixed and the same sampling pattern is applied to each sensor, yielding separable samples in the space-time domain. In coprime arrays in $2 \mathrm{D}$, sensors can be arbitrarily placed over nonseparable sampling lattices. Coprime JADE transforms separable samples to the angle-Doppler plane, which is nonseparable since $\left(\bar{\theta}_{i}, \bar{f}_{i}\right)$ can be placed anywhere on the angle-Doppler plane. Coprime arrays in 2D convert their nonseparable inputs to the azimuth-elevation domain, which is also nonseparable.

Theorem 1 suggests that $\mathbf{R}_{s s}$ can be utilized in MUSIC algorithms to estimate $\left(\bar{\theta}_{i}, \bar{f}_{i}\right)$. It is because $\mathbf{R}_{s s}$ shares the same eigenvectors as the term between the parentheses in (8). Assume the signal subspace of $\mathbf{R}_{s s}$ is represented as $\mathbf{U}_{s}$, and let $\mathbf{U}_{n}$ denote the noise subspace of $\mathbf{R}_{s s}$. Following the definition of MUSIC spectra [4], the MUSIC spectrum for coprime JADE is then defined as

$$
P_{M U S I C}(\bar{\theta}, \bar{f})=\frac{1}{\left\|\mathbf{U}_{n}^{H} \tilde{\mathbf{w}}_{s, t}(\bar{\theta}, \bar{f})\right\|_{2}^{2}},
$$

where $\bar{\theta}, \bar{f} \in[-1 / 2,1 / 2)$ and $\|\cdot\|_{2}$ denotes Euclidean norms of vectors. Then, the DOAs and the Doppler frequencies can be estimated by detecting the peaks in $P_{M U S I C}(\bar{\theta}, \bar{f})$.

\section{HOW MANY SOURCES CAN COPRIME JADE IDENTIFY?}

The number of identifiable sources for coprime JADE will be discussed in this section. Under some conditions, the MUSIC spectrum $P_{M U S I C}(\bar{\theta}, \bar{f})$ can identify up to $M_{1} N_{1} M_{2} N_{2}$ correct angleDoppler pairs.

It was shown in Section 3 that coprime JADE is closely related to nested arrays or coprime arrays in two dimensions. In 2D, identifiability issues become difficult and can be categorized into almostsure identifiability [16] and exact identifiabilty [14]. In this paper, we propose the following theorem on exact identifiability, based on the theoretical results on identifiability properties given in [14],

Theorem 2. Consider distinct sources $S=\left\{\left(\bar{\theta}_{i}, \bar{f}_{i}\right)\right\}_{i=1}^{D}$ where $D \leq M_{1} N_{1} M_{2} N_{2}$. Assume that a) the set $\left\{\bar{\theta}_{i}\right\}_{i=1}^{D}$ takes at most $M_{1} N_{1}$ distinct values and $\left.b\right)\left\{\bar{f}_{i}\right\}_{i=1}^{D}$ contains at most $M_{2} N_{2}$ distinct values. Then, $P_{M U S I C}(\bar{\theta}, \bar{f})$ has a singularity if and only if $(\bar{\theta}, \bar{f}) \in S$.

Proof. According to Theorem 4 in [14], letting $M=M_{1} N_{1}+1$ and $N=M_{2} N_{2}+1$, we obtain that $P_{M U S I C}(\bar{\theta}, \bar{f})$ has a singularity if and only if $\tilde{\mathbf{w}}_{s, t}(\bar{\theta}, \bar{f})=\tilde{\mathbf{w}}_{s, t}\left(\bar{\theta}_{i}, \bar{f}_{i}\right)$ for some $1 \leq i \leq D$. Provided with $\tilde{\mathbf{w}}_{s, t}(\bar{\theta}, \bar{f}), \tilde{\mathbf{w}}_{s}(\bar{\theta})$ is the first $M_{1} N_{1}+1$ entries of $\tilde{\mathbf{w}}_{s, t}(\bar{\theta}, \bar{f})$ and $\tilde{\mathbf{w}}_{t}(\bar{f})$ corresponds to the $\left(1+\left(M_{1} N_{1}+1\right) \ell\right)$ th entries of $\tilde{\mathbf{w}}_{s, t}(\bar{\theta}, \bar{f}), \ell=0,1, \ldots, M_{2} N_{2}$. Hence $\tilde{\mathbf{w}}_{s, t}(\bar{\theta}, \bar{f})=$ $\tilde{\mathbf{w}}_{s, t}\left(\bar{\theta}_{i}, \bar{f}_{i}\right)$ is equivalent to $\tilde{\mathbf{w}}_{s}(\bar{\theta})=\tilde{\mathbf{w}}_{s}\left(\bar{\theta}_{i}\right)$ and $\tilde{\mathbf{w}}_{t}(\bar{f})=$ $\tilde{\mathbf{w}}_{t}\left(\bar{f}_{i}\right)$ for some $1 \leq i \leq D$. Since $\tilde{\mathbf{w}}_{s}(\bar{\theta})$ and $\tilde{\mathbf{w}}_{t}(\bar{f})$ correspond to ULAs and uniform sampling, respectively, applying Theorem 1 in [17] twice implies that $\tilde{\mathbf{w}}_{s}(\bar{\theta})$ and $\tilde{\mathbf{w}}_{t}(\bar{f})$ are invertible. Hence, for some $1 \leq i \leq D, \bar{\theta}=\bar{\theta}_{i}$ and $\bar{f}=\bar{f}_{i}$, which complete the proof.

Theorem 2 indicates the maximum number of identifiable sources is $M_{1} N_{1} M_{2} N_{2}$. This result can be interpreted as a combination of coprime arrays and coprime samplers. Theories in coprime arrays proved that up to $M_{1} N_{1}$ DOAs can be detected [9]. Coprime samplers have up to $M_{2} N_{2}$ degrees of freedom [9]. If the $D$ sources exhibit $M_{1} N_{1}$ distinct values projected onto $\bar{\theta}$ and $M_{2} N_{2}$ distinct values onto $\bar{f}_{i}$, then these values are distinguishable using coprime arrays and coprime samplers. 


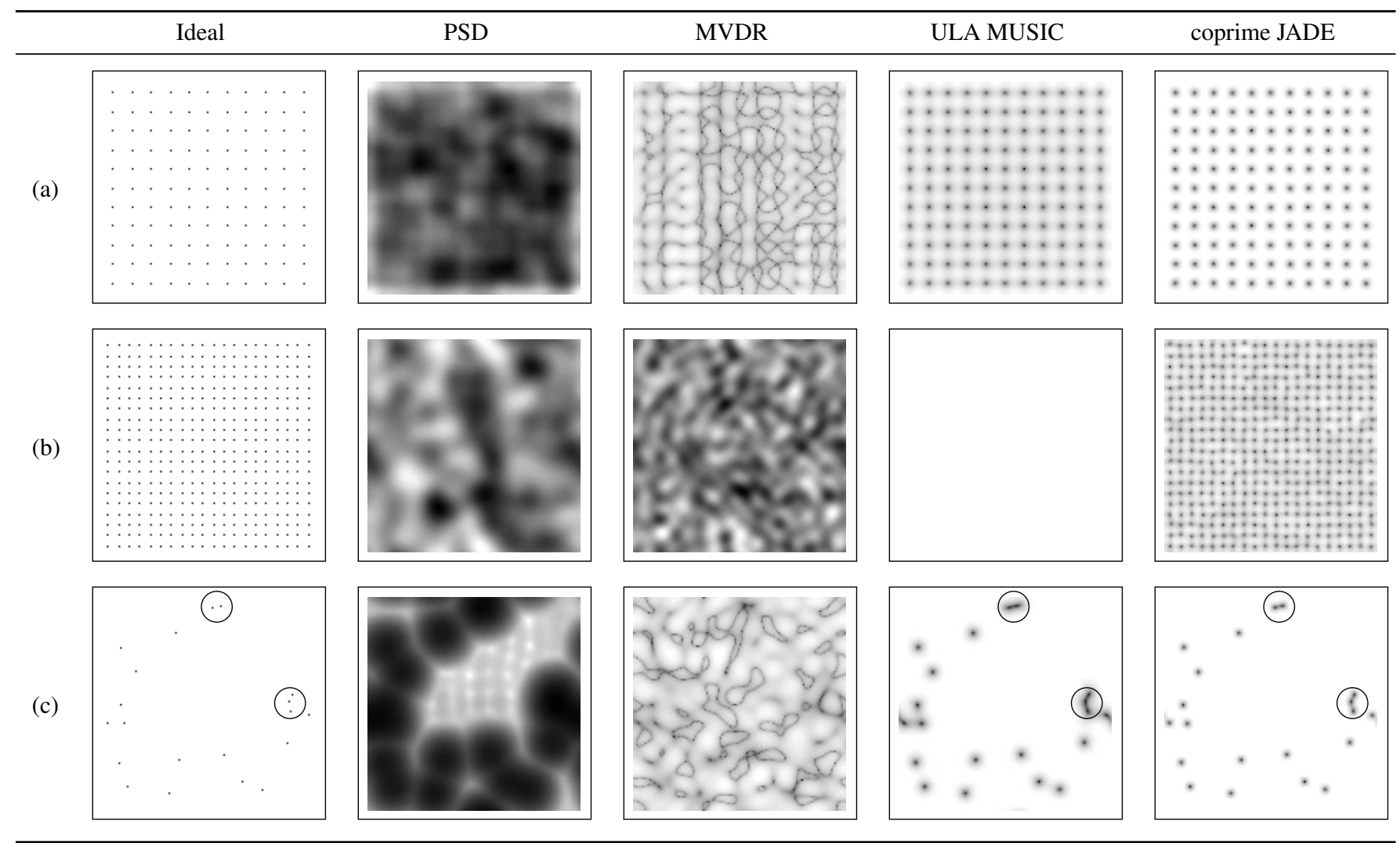

Fig. 3. Angle-Doppler pattern using different methods and different sources. (a) 121 uniform sources, (b) 400 uniform sources, and (c) 20 random sources. $x$ axes stand for normalized DOAs $\bar{\theta}$ while $y$ axes represent normalized Doppler frequencies $\bar{f}$. The color is reversed (black shows larger values) to enhance the contrast.

\section{NUMERICAL RESULTS}

The performance of different algorithms is compared in this section. We compare Fourier-based power spectrum density (PSD) [1], Minimum variance distortionless response (MVDR) [3], MUSIC algorithm with ULAs [4], and coprime JADE. The former three methods use ULAs plus uniform time samples at each sensor. In these simulations, $M_{1}=M_{2}=4$ and $N_{1}=N_{2}=5$ so that the number of sensors and the number of samples per sensor are exactly 12 in all methods. According to (5), the autocorrelation matrix of $\mathbf{x}$ is estimated from 1000 snapshots. The input signal is mixed with additive white Gaussian noise with $0 \mathrm{~dB}$ SNR. Simulation results are shown in Fig. 3.

In the first simulation, $11^{2}=121$ sources are placed uniformly on the angle-Doppler plane. This example hits the limit of ULA and uniform sampling since there are only 12 sensors and 12 samples at each sensor. PSD and MVDR fail to separate all 121 sources but MUSIC-based methods show similar patterns as the ideal case.

In the second simulation, the number of sources is increased to $20^{2}=400$, which is the maximum number of distinguishable sources for coprime JADE. The first three methods cannot detect all 400 sources. ULA MUSIC method cannot resolve the spectrum due to its rank deficiency. However, coprime JADE still works in this extreme case.

Our third example selects 20 random sources. PSD and MVDR still cannot resolve these sources while ULA MUSIC and coprime JADE work. However, as seen from the circled parts in these figures,
ULA MUSIC produces more blurry spectrum than coprime JADE, for closely spaced sources. Therefore, coprime JADE has higher resolution and is suitable for closely spaced sources.

\section{CONCLUDING REMARKS}

In this paper, we have formulated coprime JADE as a novel approach to angle-Doppler estimation, inspired by the concept of coprime arrays and coprime samplers. From the input samples, it was realized by constructing a matrix as a sum of $2 \mathrm{D}$ complex sinusoids, from which a spatial smoothed matrix was established. Theorem 1 on the spatial smoothed matrices allowed a definition of the MUSIC spectrum over the angle-Doppler plane. Coprime JADE exhibited unique identifiability up to $M_{1} N_{1} M_{2} N_{2}$ distinct sources as long as these sources satisfy some conditions. These results were verified through simulations and coprime JADE resolved closely spaced sources much better than some conventional approaches.

Further research will be directed toward the connection between coprime JADE and coprime arrays in high dimensions $[14,15]$. Coprime JADE takes the same number of samples at each sensor, which follows the same formulation as separable coprime arrays in two dimensions. It could be possible to design different sampling schemes at different sensors such that the number of samplers is reduced but the performance is unchanged. 


\section{REFERENCES}

[1] R. Klemm, Space-Time Adaptive Processing: Principles and Applications, IEEE Press, 1998.

[2] J. R. Guerci, Space-Time Adaptive Processing for Radar, Artech House, 2003.

[3] J. Capon, "High-resolution frequency-wavenumber spectrum analysis," Proc. IEEE, vol. 57, no. 8, pp. 1408-1418, Aug 1969.

[4] R. Schmidt, "Multiple emitter location and signal parameter estimation," IEEE Trans. Antennas Propag., vol. 34, no. 3, pp. 276-280, Mar 1986.

[5] R. Roy and T. Kailath, "Esprit-estimation of signal parameters via rotational invariance techniques," IEEE Trans. Acoust., Speech, Signal Process., vol. 37, no. 7, pp. 984-995, Jul 1989.

[6] Y.-Y. Wang, J.-T. Chen, and W.-H. Fang, "TST-MUSIC for joint DOA-delay estimation," IEEE Trans. Signal Process., vol. 49, no. 4, pp. 721-729, Apr 2001.

[7] D. D. Ariananda and G. Leus, "Compressive joint angularfrequency power spectrum estimation," in Proc. the 21st European Signal Processing Conference (EUSIPCO 2013), Marrakech, Morocco, Sep. 2013.

[8] P. P. Vaidyanathan and P. Pal, "Sparse sensing with co-prime samplers and arrays," IEEE Trans. Signal Process., vol. 59, no. 2, pp. 573-586, Feb 2011.

[9] P. Pal and P. P. Vaidyanathan, "Coprime sampling and the MUSIC algorithm," in Proc. IEEE Digital Signal Processing Workshop and IEEE Signal Processing Education Workshop, Jan 2011, pp. 289-294.
[10] Y. Zhang, M. Amin, and B. Himed, "Sparsity-based DOA estimation using co-prime arrays," in Proc. IEEE Int. Conf. Acoust., Speech, and Sig. Proc., May 2013, pp. 3967-3971.

[11] T.-J. Shan, M. Wax, and T. Kailath, "On spatial smoothing for direction-of-arrival estimation of coherent signals," IEEE Trans. Acoust., Speech, Signal Process., vol. 33, no. 4, pp. 806811, Aug 1985.

[12] Y.-M. Chen, "On spatial smoothing for two-dimensional direction-of-arrival estimation of coherent signals," IEEE Trans. Signal Process., vol. 45, no. 7, pp. 1689-1696, Jul 1997.

[13] P. Pal and P. P. Vaidyanathan, "Nested arrays: A novel approach to array processing with enhanced degrees of freedom," IEEE Trans. Signal Process., vol. 58, no. 8, pp. 4167-4181, Aug 2010.

[14] P. Pal and P. P. Vaidyanathan, "Nested arrays in two dimensions, part II: Application in two dimensional array processing," IEEE Trans. Signal Process., vol. 60, no. 9, pp. 47064718, Sept 2012.

[15] P. P. Vaidyanathan and P. Pal, "Theory of sparse coprime sensing in multiple dimensions," IEEE Trans. Signal Process., vol. 59, no. 8, pp. 3592-3608, Aug 2011.

[16] T. Jiang, N. Sidiropoulos, and J. ten Berge, "Almost-sure identifiability of multidimensional harmonic retrieval," IEEE Trans. Signal Process., vol. 49, no. 9, pp. 1849-1859, Sep 2001.

[17] P. P. Vaidyanathan and P. Pal, "Direct-MUSIC on sparse arrays," in 2012 International Conference on Signal Processing and Communications (SPCOM), July 2012. 